index) or z-score WFH (weight for height) from the time of admission to discharge. Logistic regression analysis was performed to determine risk factors of HAM.

Results We included 294 children in the study, with a mean age of 43.6 months. Conditions affecting upper and lower respiratory tract $(32 \%)$ were the most common at admission followed by infectious diseases $(21 \%)$ and gastrointestinal system conditions $(10.5 \%)$. The prevalence of acute undernutrition (AUN) at admission was $25.5 \%$. It was mild, moderate and severe respectively in $17 \%, 5 \%$ and $3.5 \%$ of cases. The prevalence of AUN at discharge was $34 \%$. It was mild, moderate and severe respectively in $19 \%, 8 \%$ and $7 \%$ of cases. Duration of hospital stay ranged from 2 to 41 days with a median of 5 days. The prevalence of HAM was 28.6\% (84/294) with a predominance in children aged $<59$ months (68/205) (33.2\%). Weight loss was observed in 63.2\% (186/294) of cases. The mean weight at admission was $15.7 \pm 12.9 \mathrm{kgs}$ [2.2-80]. At discharge, the mean weight dropped to 15.3 $\pm 12.8 \mathrm{kgs}$ [1.6-77]. All age groups showed a reduction in weight during hospitalization; however, it was more common in children aged 12-24 months (61.8\%). Weight loss was $\geq 5 \%$ in $82 / 186(44 \%)$ of cases. Risk factors of HAM were : separation from mother during hospitalization (Odds Ratio (OR)3.44, 95\%CI 1.13-10.48; p=0.029), fever during hospitalization (OR 8.94, CI 1.1-72.42; $\mathrm{p}=0.04$ ), stop or decrease oral or enteral feeding during hospitalization (OR 1.47, CI 1.27-1,82; $\mathrm{p}=0.008)$. Howerver, breastfeeding, absence of chronic disease and age over 15 months were protective factors against HAM.

Conclusion The prevalence of HAM in Tunisian children was high. Risk factors are multiple and their screening is essential for early and adequate management.

\section{P514 LATCHON: A MULTI-CENTRE, RANDOMISED CONTROLLED TRIAL OF PERINATAL SUPPORT TO IMPROVE BREASTFEEDING OUTCOMES IN WOMEN WITH OVERWEIGHT AND OBESITY}

\begin{abstract}
'Eileen O'Brien*, 'Sharleen O'Reilly, ${ }^{2}$ Lucille Sheehy, ${ }^{2}$ Lorraine O'Hagan, ${ }^{1}$ Denise McGuinness, ${ }^{1}$ Barbara Coughlan, 'Denise O'Brien, ${ }^{2}$ Rosie Murtagh, ${ }^{3}$ Marie Corbett, ${ }^{3}$ Michelle Walsh, ${ }^{4}$ Paula Power, ${ }^{4}$ Marie Woodcock, ${ }^{4}$ Amy Carroll, ${ }^{5}$ Stephanie Murray, ${ }^{5}$ Charmaine Scallan, ${ }^{5}$ Elizabeth Dunne, ${ }^{1}$ Fionnuala McAuliffe. ${ }^{1}$ University College Dublin, Dublin, Ireland; ${ }^{2}$ National Maternity Hospital, Dublin, Ireland; ${ }^{3}$ Regional Hospital Mullingar, Westmeath, Ireland; ${ }^{4}$ St.Luke's General Hospital, Kilkenny, Ireland; ${ }^{5}$ Wexford General Hospital, Wexford, Ireland
\end{abstract}

\subsection{6/archdischild-2019-epa.850}

Background Breastfeeding rates in Ireland are among the lowest worldwide. At hospital discharge, $58 \%$ of infants are breastfed, with only $48 \%$ exclusively breastfed. At 3 months of age, 35\% are fed any breastmilk. Women with a high BMI have lower initiation rates and duration of breastfeeding, which is a particular concern in Ireland given that $50 \%$ of women have a BMI of $>25 \mathrm{~kg} / \mathrm{m}^{2}$ at their first antenatal appointment.

Objective The aim of the intervention is to improve breastfeeding rates using a previously-tested, multi-component intervention. The intervention will target attitudes toward breastfeeding, breastfeeding self-efficacy, and subjective norms around infant feeding with the aim of normalising the behaviour.

Methods This protocol is for a multi-centre, randomised controlled trial of perinatal breastfeeding support among women with a BMI $>25 \mathrm{~kg} / \mathrm{m}^{2}$. Hospital discharge data, validated questionnaires and qualitative interviews will be used to measure outcomes and intervention effectiveness. Ethical approval has been sought and recruitment will commence in early 2019. Patients: Primiparous women attending the study site hospitals with a singleton pregnancy and BMI $>25 \mathrm{~kg} / \mathrm{m}^{2}$.

Intervention The intervention will target mothers and their support partners and will span the perinatal period from late pregnancy to six weeks postpartum. Intervention components include: group antenatal education for prospective mothers and their support partners; individual education in the immediate postnatal period; professional support to six weeks postpartum; and weekly phone calls in the postpartum period from an International Board-Certified Lactation Consultant. The primary outcome is prevalence of breastfeeding at 3 months.

Results We anticipate that the intervention will be wellaccepted and feasible to carry out within an Irish cohort based on results from the pilot trial among 100 women. Furthermore, essential formative qualitative work has been conducted to inform the intervention design and to ensure that it is contextually appropriate.

Conclusions The proposed intervention will be invaluable to policy-makers as it will provide insights into the specific interventions (e.g. antenatal group education, antenatal individual education, postpartum support) that are effective in improving breastfeeding rates for women with a raised BMI and will highlight the measures that would be most cost-effective to implement nationally.

\section{P516 THE POSITIVE CLINICAL CONSEQUENCE OF EARLY INTERVENTION OF COMBINED THERAPY (OMEGA 3 FATTY ACIDS AND B12 VITAMIN) ON CHILDREN UNDER 5 WITH VARIABLE FORMS OF CEREBRAL PALSY}

Khajik Yaqob*. Zakho General Hospital/Kurdistan Pediatrics Society, DUHOK, Iraq

\subsection{6/archdischild-2019-epa.851}

Background Cerebral palsy is a common pediatric problem encountered in about 1:3 per 1000 born children and causing variable mental, motor and behavioral s dilemmas. Newly introduced trials of neurogenesis with different agents are now extensively evaluated.

Objective Our study was conducted to evaluate the neurotrophic response to B12 vitamin and omega-3 fatty acids in children diagnosed early with variable forms of cerebral palsy. The response was monitored both clinically and with C.T Scan as being a highly predictive tool for assessing cerebral palsy.

Design The study was carried out on 40 cerebral palsy patients; $26(65 \%)$ out of them were girls, and 14 of them were boys, aged from 0 to 5 years old; from outpatient clinic at Zakho/Duhok General Hospital in Kurdistan Region-Iraq. Patients were treated and followed up for 6 month to one year. They were represented and adjusted by full history taking and clinical examination. Brain C.T scans was done for every patient to assess the degree of brain atrophy before starting this combined therapy, and every month for six months to one year.

Results The study revealed that early intervention of both omega 3 and B12 vitamin in children under 5 with cerebral palsy (cp) shows great response based on clinical examination 
and CT scan findings. after combined therapy, $80 \%$ of children with delayed speech delay have very good response and improvement, $77 \%$ of children with delayed milestone and hypertonia, and $87 \%$ with delayed walking have positive clinical outcomes. Both sexes have equal response to combined therapy. Such findings were obtained as a result of early treatment and diagnosis of children with (CP). In addition, improvement in CT scan results was obtained. $84 \%$ of treated children have great improvement in their neuroimaging results from moderate/severe forms of brain atrophy to mild form of brain atrophy after being treated and followed up for 6 months- 1 year.

Conclusions The damaged brain sites based on CT scan results, showed progressive improvement in response to B12 and omega-3 fatty acids upon daily supplement throughout 6 months to one year. B12 vitamin and omega- 3 fatty acids are valuable therapy for children with various forms of cerebral palsy particularly when being linked. The greatest improvement in speech and motor development was significantly observed in about 32 patients (80\%) of treated children with combined therapy. Others have less response to combine therapy as being presented and diagnosed beyond 1 year of age $(16 \%)$

\section{P517 METABOLIC FEATURES OF ADOLESCENTS WITH OBESITY}

Lyubov Rychkova*, Anna Pogodina, Lyudmila Grebenkina, Olga Berdina, Zhanna Ayurova, Tatyana Astakhova. Scientific Centre for Family Health and Human Reproduction Problems, Irkutsk, Russian Federation

\subsection{6/archdischild-2019-epa.852}

Introduction Over the last decades researchers discuss the interconnection between obesity and metabolic syndrome, the main manifestations of which include obesity, dyslipidemia, insulin resistance, and hypertension.

Aim To determine biochemical parameters in obese adolescents of Asian and Caucasian ethnicities, living in a rural area.

Materials and methods We investigated 200 adolescents with overweight and obesity aged 11-17 years: 43 Asian adolescents and 29 Caucasian adolescents. The control group comprised 128 adolescents with normal body weight in both ethnic groups. All adolescents were living in Buryatia (Russia). Overweight was diagnosed when body mass index (BMI) $>85$ percentile, obesity - at BMI $\geq 95$. Metabolic status was established by the spectrophotometric method. The following parameters were determined and analyzed: total cholesterol, high and low density lipoproteins fractions, triglycerides, fasting glucose and two-hour post-load glucose levels.

Results After comparison of biochemical parameters, we found changes in carbohydrate metabolism in obese adolescents. Glucose tolerance test showed significant differences between twohour post-load glucose levels between groups with obesity and normal body weight $(4.63 \pm 0.62 \mathrm{mmol} / \mathrm{L}$ against $3.89 \pm$ $0.62 \mathrm{mmol} / \mathrm{L}$, respectively in Asian adolescents, $\mathrm{p}=0.000$ $4.48 \pm 0.9 \mathrm{mmol} / \mathrm{L}$ against $3.89 \pm 0.5 \mathrm{mmol} / \mathrm{L}$, respectively in Caucasian adolescents, $p=0.000$ ). We found significant differences between levels of this parameter among adolescents with obesity of Asian and Caucasian ethnicities (4.63 \pm 0.62 $\mathrm{mmol} / \mathrm{L}$ against $4.48 \pm 0.9 \mathrm{mmol} / \mathrm{L}$, respectively, $\mathrm{p}=0.006)$. Mean group values of fat metabolism in adolescents with obesity were within normal values, however during a frequency analysis we detected changes: hypercholesteremia in $6.8 \%$ in
Asian adolescents and 4.6\% in Caucasian adolescents. Hypertriglyceridemia was detected in $16.3 \%$ cases of Asians and $9.2 \%$ in the Caucasian group, $\mathrm{p}=0.007$. Hypolipoproteinemia in obese adolescents was noticed in $6.7 \%$ of Asians (in Caucasian group $-3.4 \%, \mathrm{p}=0.569$ ).

Conclusions Therefore all metabolic changes, mentioned above, are more typical for the natives than for newcomer, namely, Caucasian people, which probably may be associated with food stereotypes and consuming food rich in animal fats. At the same time traditional food stereotypes in Asian ethnicities may contribute to adaptation to atherogenesis and development of resistance to atherosclerosis and diabetes mellitus, predominantly of the type 1 .

\section{P518 FUNCTIONAL DISORDERS OF THE COLON IN ADOLESCENTS WITH OBESITY: PSYCHOLOGICAL CHARACTERISTICS}

Anastasiya Romanitsa, Lyubov Rychkova*, Anna Pogodina. Scientific Centre for Family Health and Human Reproduction Problems, Irkutsk, Russian Federation

\subsection{6/archdischild-2019-epa.853}

Adolescent obesity is a very serious problem of the 21 century. Approximately $86.5 \%$ of obese adolescents have functional disorders of the colon (FDC), among which the most prevalent disorder is irritable bowel syndrome (IBS) - 76.1\%. One of the causes of FDC is a psychological state. Psychological problems, may develop into more serious conditions, such as severe depression, low self-esteem, anorexia nervosa, and bulimia nervosa.

Aim To determine psycho-emotional disorders in adolescents with obesity and FDC.

Materials and methods We examined 111 children with obesity (11-17 years), among them 64 girls and 47 boys, age 14.5 \pm 2.0 , over a period 2016-2018. Depending on the presence of FDC in adolescents with obesity, the sample was divided in 3 groups: 1 group included $73(65.8 \%)$ adolescents with obesity and IBS (diagnosed according to The Rome IV criteria), 2 group - $23(20.7 \%)$ adolescents with obesity and other FDC, 3 group - 15 (13.5\%) adolescents with obesity without FDC. We conducted a questionnaire survey on the psychoemotional state of adolescents (State-Trait Anxiety Inventory, Rosenberg's Faith in People scale, Beck Depression Inventory, EAT-26). Results were processed using software Statistica 10.0 for Windows. The difference was considered significant at $\mathrm{p}<0.05$. Results EAT-26 did not show differences between groups on the following indicators: pursuance of leanness, bulimia, inefficiency, interoceptive incapacity. There were significant differences in the group with IBS $(p=0.000)$ on discomfort with one's body, distrust in interpersonal relationships. Rosenberg's Faith in People scale did not show differences between groups $(p=1.2)$. State-Trait Anxiety Inventory showed that state anxiety and trait anxiety are more pronounced in groups with IBS and other FDC. There is a significant difference between groups with IBS and other FDC and the group without FDC $(p=0.000)$. Depression is significantly higher in the group with IBS $(p<0.001)$ according to Beck Depression Inventory.

Conclusions We found maladaptive eating behavior (pursuance for leanness, bulimia, inefficiency, interoceptive incapability) in adolescents with obesity, regardless of FDC. We observed increased discomfort with the body, distrust in interpersonal 